\title{
Atendimento à Mulher vítima de violência sexual: Protocolo de Assistência de Enfermagem
}

\author{
ASSISTANCE TO WOMEN VICTIMS OF SEXUAL VIOLENCE: A NURSING CARE PROTOCOL \\ ATENCIÓN A LA MUJER VÍCTIMA DE VIOLENCIA SEXUAL: \\ PROTOCOLO DEASISTENCIA DE ENFERMERÍA
}

\author{
Rosângela Higa ${ }^{1}$, Aurélia Del Carmen Alvarez Mondaca ${ }^{2}$, Maria José dos Reis ${ }^{3}$, \\ Maria Helena Baena de Moraes Lopes ${ }^{4}$
}

\section{RESUMO}

O M inistério da Saúde preconiza o atendimento integral e humanizado às mulheres vítimas de violência sexual. Teve-se por objetivo descrever o Protocolo de Enfermagem na Assistência às Mulheres Vítimas de Violência Sexual do Centro de Atenção Integral à Saúde da M ulher da Universidade Estadual de Campinas, recentemente revisado. Para tanto, seguiram-se as etapas do processo de enfermagem e após a identificação dos principais diagnósticos da North American Nursing Diagnosis Association (NANDA) foram determinadas as intervenções, com base em normas técnicas nacionais e internacionais. 0 protocolo atual engloba 0 atendimento imediato $e$ tardio, o acompanhamento ambulatorial e as ações relacionadas à interrupção legal da gravidez decorrente do estupro. 0 protocolo de enfermagem tem proporcionado à cliente um atendimento integral e humanizado e à enfermeira, maior autonomia na sua área de atuação, favorecendo o trabalho colaborativo e interativo com a equipe multidisciplinar.

\section{DESCRITORES}

Saúde da mulher.

Cuidados de enfermagem.

Violência sexual.

Diagnóstico de enfermagem.

\begin{abstract}
The M inistry of Health recommends integral and humanized assistance to women victims of sexual violence. This study was aimed at describing the Nursing Protocol in the Attention to Women Victims of Sexual Violence at the Center for Integral Attention to Women's Health of the State University of Campinas (Unicamp), which has recently been revised. To do so, the phases of the nursing process were followed, and after the identification of the main nursing diagnoses of the North American Nursing Diagnosis Association (NANDA) the nursing interventions were determined, based on international and national guidelines care for sexual violence. The current protocol encompasses both immediate and late care, outpatient follow-up and actions regarding legal interruption of pregnancy resulting from rape. The nursing protocol has been providing integral and humanized assistance to women and, for nurses, more autonomy in their area and the possibility of participative and collaborative work with multidisciplinary teams.
\end{abstract}

\section{KEY WORDS}

Women's health. Nursing care.

Sexual violence.

Nursing diagnosis.

\section{RESUMEN}

El M inisterio de Salud preconiza la atención integral y humanizada a las mujeres víctimas de violencia sexual. Se tuvo como objetivo describir el Protocolo de Enfermería en la Asistencia a las Mujeres Víctimas de Violencia Sexual del Centro de Atención Integral a la Salud de la M ujer de la Universidad Estatal de Campinas, recientemente revisado. Para tal efecto, se siguieron las etapas del proceso de enfermería y después de la identificación de los principales diagnósticos de la North American Nursing Diagnosis Association (NANDA) fueron determinadas las intervenciones, con base en normas técnicas nacionales e internacionales. El protocolo actual engloba la atención inmediata y tardía, el acompañamiento ambulatorio y las acciones relacionadas a la interrupción legal del embarazo derivado de la violación. El protocolo de enfermería ha proporcionado a la cliente una atención integral y humanizada y a la enfermera, mayor autonomía en su área de actuación, favoreciendo el trabajo colaborador e interactivo con el equipo multidisciplinario.

\section{DESCRIPTORES}

Salud de la mujer.

Atención de enfermería.

Violencia sexual.

Diagnóstico de enfermería.

\footnotetext{
${ }^{1}$ Enfermeira. Mestre em Enfermagem. Supervisora da Divisão de Enfermagem do Centro de Atenção Integral a Saúde da Mulher da Universidade Estadua de Campinas (Caism/Unicamp). Campinas, SP, Brasil. ro-higa@ig.com.br ${ }^{2}$ Enfermeira. Especialista em Enfermagem Obstétrica da Divisão de Enfermagem do Caism/Unicamp. Campinas, SP, Brasil. aureliaalvarez@superig.com.br ${ }^{3}$ Enfermeira. Especialista em Ginecologia. Supervisora da Divisão de Enfermagem do Caism/Unicamp. Campinas, SP, Brasil. mjreis03@hotmail.com ${ }^{4}$ Enfermeira Livre-docente. Professora Associada do Departamento de Enfermagem da Faculdade de Ciências Médicas da Unicamp. Campinas, SP, Brasil. mhbaena@fcm.unicamp.br
} 


\section{INTRODUÇÃO}

No Brasil, assim como em diversos países do mundo, a violência sexual constitui um sério problema de saúde pública por ser uma das principais causas de morbidade e mortalidade feminina ${ }^{(1)}$. A violência baseada em questões de gênero é também uma violação dos direitos humanos. Acomete mulheres de todas as idades, de diferentes níveis econômicos e sociais, em espaço público ou privado e em qualquer fase de sua vida(2).

Nos serviços de saúde, a mulher violentada sexualmente necessita de acolhimento ${ }^{(2)}$, fator fundamental para a humanização da assistência à saúde e essencial para que se estabeleça um relacionamento de forma adequada entre o profissional e a cliente $\mathrm{e}^{(3)}$.

Nos Estados Unidos e Canadá, os programas de Sexual Assault Nurse Examiners (SANE - Enfermeiras Examinadoras de Agressão Sexual), nos quais a enfermeira é especialista na realização do exame forense em crianças e adultos, têm mostrado eficientes resultados em reduzir o trauma decorrente da agressão sexual na avaliação e tratamento às vítimas de violência sexual ${ }^{(4-5)}$. Portanto, a enfermeira deve estar habilitada para acolher e desenvolver assistência voltada para a recuperação física, psicológica e social, sem demonstrar atitudes preconceituosas ${ }^{(2)}$ que possam interferir na adesão ao tratamento.

O Centro de Atenção Integral à Saúde da Mulher da Universidade Estadual de Campinas (Caism/Unicamp), hospital de ensino especializado na assistência à saúde da mulher e do recém-nascido, é um dos serviços pioneiros no país a prestar assistência nos casos de gravidez decorrente de estupro (aborto legal, pré-natal e processo de doação). Esse hospital, desde 1998, dispõe de equipe treinada para assistir à mulher vítima de violência sexual com protocolos multidisciplinares que visam prevenir a gravidez, as doenças sexualmente transmissíveis (DST), incluindo o vírus da imunodeficiência humana (HIV), e promover a recuperação física, psicológica e social da mulher. A equipe multidisciplinar do Caism, além de capacitar os funcionários das diferentes unidades de atendimento, portaria, serviço de arquivo médico e outros, tem promovido cursos, palestras em nível regional, estadual e nacional com a finalidade de treinar profissionais de saúde para este atendimento.

o Caism participa do Projeto lluminar Campinas: Cuidando das Vítimas de Violência Sexual(6), programa da Secretaria Municipal de Saúde de Campinas, implantado em 2001, em parceria com o M inistério da Saúde. Esse projeto atua com uma rede intersetorial e interinstitucional de serviços (órgãos governamentais e não governamentais locais) nas áreas de saúde, educação, assistência social, jurídica e de cidadania, e visa cuidar da saúde física, mental, social e civil de todas as vítimas de violência sexual urbana ou doméstica, independente de sexo e idade; prevenir a gravidez por estupro e as DST virais e não virais; intervir na cadeia de violência por meio do cuidado às pessoas autoras de agressão, em ambiente não policial, e utilizar o banco de dados, dos casos notificados, para criar indicadores gerenciais e epidemiológicos da violência sexual no município.

As enfermeiras, por atuarem 24 horas dentro da instituição, foram consideradas as profissionais adequadas para realizar 0 acolhimento ${ }^{(7)}$. Contudo, era preciso adquirir conhecimento sobre os aspectos assistenciais e legais que envolvem a violência sexual, bem como treinar a equipe de enfermagem de forma a sensibilizar e capacitar seus membros para acolher, oferecer conforto e segurança à mulher. Para tanto, buscou-se na revisão da literatura e contato com o SOS Ação Mulher e Família e Delegacia da Mulher de Campinas, instituições que prestam este tipo de atendimento, informações necessárias para delinear a assistência a essas mulheres, visto que nesta época as Normas sobre a Prevenção e Tratamento dos Agravos Resultantes da Violência Sexual contra Mulheres e Adolescentes(2), do Ministério da Saúde, ainda não haviam sido publicadas.

O SOS Ação Mulher e Família(8), fundado em 1980, é uma organização não-governamental sediada em Campinas, SP. Atualmente constitui-se como entidade de Utilidade Pública M unicipal, Estadual e Federal e mantém, desde 1987, convênio de cooperação com a Unicamp.

Perante 0 interesse em acolher estas mulheres, um grupo de enfermeiras do Caism, em conjunto com assistentes sociais e psicólogas, elaborou, em agosto de 1998, o protocolo intitulado Atendimento Multidisciplinar às Mulheres Vítimas de Violência Sexual(7), que inclui o Protocolo de Atendimento de Enfermagem. A utilização do protocolo de enfermagem norteou o cuidar, facilitou o processo de enfermagem e garantiu o registro adequado das intervenções. Em decorrência disto, a assistência de enfermagem prestada contribuiu para que as mulheres atendidas continuassem a viver com dignidade na busca dos restabelecimentos físico e psicológico afetados pela violência.

Após seis anos de implantação do protocolo multidisciplinar, novos programas surgiram como 0 atendimento tardio e o seguimento ambulatorial. 0 protocolo vigente não descrevia a atuação da enfermeira e dos demais profissionais nestes programas e, além disso, houve implantação da ficha única de atendimento multidisciplinar e ocorreram alterações no protocolo médico, tais como a inclusão de novos medicamentos na quimio profilaxia para DST/HIV(9-11) e utilização da anticoncepção de emergência $(\mathrm{AE})^{(12-13)}$ até cinco dias após a agressão sexual. Esses fatos levaram a uma revisão do protocolo por cada grupo profissional envolvido nesse programa.

A revisão da literatura apontou que após a publicação das Normas Técnicas do Ministério da Saúde ${ }^{(2)}$, muitos serviços de saúde têm oferecido atendimento multidisciplinar às mulheres vítimas de violência sexual, no entanto, as atribuições da enfermeira parecem não estar 
bem estabelecidas, uma vez que o M inistério da Saúde não definiu o papel da enfermeira nesta assistência ${ }^{(14)}$.

Portanto, o objetivo deste artigo é descrever o Protocolo de Enfermagem na Assistência às Mulheres Vítimas de Violência Sexual do Caism/Unicamp já revisado, englobando 0 atendimento imediato e tardio, 0 acompanhamento ambulatorial e as ações relacionadas à interrupção legal da gravidez decorrente do estupro.

\section{MÉTODO}

0 Protocolo do Atendimento de Enfermagem às M ulheres Vítimas de Violência do Caism/Unicamp foi revisado e atualizado em janeiro de 2005, seguindo as etapas do processo de enfermagem: coleta de dados, diagnóstico, planejamento (intervenções), implementação e evolução.

A partir da identificação dos principais diagnósticos da Associação Norte Americana de Diagnósticos de Enfermagem $^{(15)}$ (NANDA - North American Nursing Diagnosis Association) foram determinadas as intervenções, adotando as sugestões do Grupo de Atendimento Multidisciplinar às M ulheres Vítimas de Violência Sexual do Caism, as recomendações das Normas Técnicas do Ministério da Saúde ${ }^{(11,13)}$ e dos protocolos da Organização Mundial de Saúde: Guidelines for M edico-legal Care for Victims of Sexual Violence (Diretrizes de Assistência M édico-Legal às Vitimas de Violência Sexual)(9) e Clinical M anagement of Rape Survivors ${ }^{(10)}$ (Gerenciamento Clínico para Sobreviventes de Estupro).

\section{RESULTADOS E DISCUSSÃO}

0 atendimento às mulheres vítimas de violência sexual compreende toda mulher pós-púbere, menopausada e não púbere maior de 14 anos que relate ter sido vítima de violência sexual das seguintes formas: estupro, atentado violento ao pudor com penetração oral e/ou anal ou sem penetração com ejaculação externa próxima à região genital; é oferecido durante as 24 horas; priorizado no momento em que a cliente chega ao serviço e em local privativo e tranqüilo(7).

A equipe multidisciplinar é composta por enfermeiros(as), ginecologistas, infectologista, assistentes sociais e psicólogas com protocolos específicos para cada área. Deve-se abrir prontuário para registro e arquivo do atendimento e observações dos diferentes profissionais, anotadas em uma única ficha clínica.

A abertura do Boletim de Ocorrência (B.O.) é prerrogativa da mulher, ela ou seus representantes legais são estimulados a comunicar às autoridades policiais e judiciárias, porém cabe a eles a decisão final; deve-se informar que a consulta ginecológica não substitui o Exame de Corpo de Delito. O hospital somente comunicará a violência às autoridades nos casos previstos em lei e ao Conselho
Tutelar da Infância e Juventude os menores de 18 anos. Todos os casos atendidos são notificados, on line, no Sistema de Notificação de Violência de Campinas (SISNOV) ${ }^{(6)}$.

Embora seja um atendimento clínico o caráter legal não pode ser ignorado, portanto, nunca se deve afirmar ou descartar a violência sexual perante a cliente e recomenda-se a presença de um profissional de enfermagem do sexo feminino, durante todo 0 atendimento, principalmente durante a consulta ginecológica ${ }^{(7)}$.

É oportuno comentar que um enfermeiro (do sexo masculino) participa do atendimento a vítimas de violência sexual e sua atuação tem sido elogiada pelas mulheres atendidas. Portanto, o profissional de saúde estando adequadamente capacitado, independente do seu sexo, poderá prestar uma assistência de qualidade.

Para 0 atendimento, a equipe de enfermagem recebe capacitação periódica e participa de oficinas de apoio psicológico (realizado pela equipe responsável pelo programa) para agir de forma imparcial, sem preconceitos, sem fazer julgamentos, sem atuar de forma comiserativa e conhecer as implicações legais, somáticas, psicológicas e sociais da violência sexual e colaborar para que a mulher não tenha que repetir várias vezes a sua história ${ }^{(11)}$.

A enfermeira acolhe, realiza triagem e faz encaminhamentos de acordo com a avaliação do tipo de violência: no caso de violência física e/ou psicológica em mulher, criança e adolescente e/ou violência sexual em menor de 14 anos não púbere, deve-se encaminhar ao pronto-socorro adulto ou pediátrico. É preciso atentar para os casos em que a menor de 18 anos confirma o consentimento da relação sexual, porém, se o responsável legal considerar que a menor não estava apta para consentir 0 ato, deve-se realizar atendimento de violência sexual.

Recomenda-se desenvolver as etapas do processo da seguinte maneira ${ }^{(10-11)}$ :

1.Por meio de entrevista coletar os seguintes dados: identificação pessoal (idade, raça, religião e outros), antecedentes ginecológicos e obstétricos, relato do ocorrido e identificação do risco de exposição à DST/HIV e gravidez. No seguimento ambulatorial, deve-se ainda: questionar o uso correto, sintomas de intolerância ou de intoxicação aos antiretrovirais (ARV), sinais e sintomas de infecção geniturinária e de gravidez; investigar aspectos emocionais, sociais e sexuais (incluindo a opção sexual); apoiar a família ou pessoa significativa. Evitar questionamentos sobre a violência sofrida e/ ou sobre a decisão de interrupção legal da gestação. A entrevista deve ser realizada, utilizando linguagem clara, com perguntas objetivas, respeitando o direito da cliente em responder ou não às perguntas e em relatar ou não o ocorrido, isentando-se de fazer julgamentos e de ter atitudes preconceituosas.

2.0bservar 0 aspecto físico geral (presença de lesões), emocional e nível de consciência. Algumas vítimas são obrigadas pelo violentador a fazer uso de drogas ilícitas, 
sendo necessário aguardar que cesse o efeito da droga antes de proceder ou continuar a entrevista. Os exames físico completo e ginecológico serão realizados pelo ginecologista. Nos casos de internação e aborto legal, a enfermeira deve realizar o exame físico, no momento da evolução da cliente, a cada 24 horas.

3. Identificar os possíveis diagnósticos e intervenções de enfermagem. Prescrever de acordo com os diagnósticos identificados para a cliente e familiares/acompanhantes. Fazer as anotações atentando ao caráter legal deste documento. É importante letra legível, tendo o cuidado em escrever as palavras: refere, informa, narra e atribui, para que fique claro que está se registrando o relato da cliente e não impressões pessoais.

De acordo com a literatura pertinente e nossa experiência profissional, considera-se que os principais diagnósticos de enfermagem são, dentre os aprovados pela NANDA, versão 2003-2004(15): Síndrome do trauma do estupro; Síndrome pós-trauma; Dor aguda; Risco para infecção; Integridade da pele prejudicada; Náusea; Conflito de decisão; Risco para angústia espiritual; Sentimento de impotência; Nutrição desequilibrada: menos do que as necessidades corporais; Nutrição Desequilibrada: mais do que as necessidades corporais; Isolamento social; Controle familiar do regime terapêutico; Controle ineficaz do regime terapêutico e Padrões de sexualidade ineficazes.

A seguir são apresentadas as principais intervenções de enfermagem de acordo com o tipo de atendimento:

Atendimento na ocorrência imediata (até 0 50 dia após a violência sexual):

1. Explicar os riscos de gravidez, infecção por DST e HIV e prevenção disponíveis, como a eficácia da $A E$ e quimioprofilaxia para DST/HIV, ressaltando que quanto mais precoce 0 início do tratamento, mais eficaz ${ }^{(10,12-13)}$.

2. Preparar para consulta médica ${ }^{(10)}$ exame físico e ginecológico com possível coleta de material forense (coleta de esperma em papel filtro).

3. Informar a equipe médica sobre os dados relevantes coletados durante a consulta de enfermagem.

4. Explicar sobre os medicamentos prescritos, a sua indicação e o tempo de tratamento(10-13): $A E$, quimioprofilaxia para DST, ARV, vacina e gamaglobulina para hepatite $B$.

5. Orientar sobre a coleta de sangue para sorologias ${ }^{(10-11)}$ de HIV, sífilis, hepatite B e C.

6. Coletar sorologias, administrar medicamentos profiláticos, vacina e gamaglobulina.

7. Encaminhar ao banho e oferecer troca de roupa, se a cliente desejar.

8. Explicar que, na ocorrência de vômito até duas horas após a ingestão do $\mathrm{AE}$, deverá repetir a dose do medicamento ${ }^{(10-11)}$.
9. Iniciar os ARV imediatamente e adequar horário, de acordo com a rotina da cliente, com objetivo de maior adesão ao tratamento.

10. Orientar os sintomas de intolerância aos ARV prescritos (manifestações gastrintestinais, cutâneas e gerais)(11).

11. Orientar meios para minimizar náuseas ou vômito.

12. Orientar hiper-hidratação oral e alimentação adequada para minimizar os efeitos colaterais dos ARV (nefropatia, anemia, hepatopatia) $)^{(11)}$.

13. Explicar a importância do uso de preservativo, por seis meses, em razão do risco de transmissão de DST/HIV e como método anticoncepcional (MAC). Orientar como usar e fornecer até o retorno ambulatorial.

14. Orientar cuidados com ferida, se apresentar lesões.

15. Orientar a observação de sintomas e manifestações clínicas de infecções geniturinárias.

16. Acolher e orientar familiares e/ ou acompanhantes com objetivo de que os mesmos proporcionem apoio no convívio diário.

17. Fornecer endereço da Delegacia de Polícia, estimular a realização do B.O. e orientar sobre a Comunicação de Acidente de Trabalho (CAT) se ocorrer durante e/ ou no percurso do trabalho(11).

18. Fornecer embalagem e orientar a guarda das roupas, em saco de papel, para ser encaminhado, pela vítima, ao Instituto Médico Legal(9).

19. Encaminhar para atendimento social e psicológico e agendar retorno ambulatorial com a enfermeira orientando sobre seguimento com equipe multidisciplinar.

Atendimento tardio (a partir do 60 dia após a ocorrência da violência sexual):

1. Explicar sobre os riscos de gravidez e de DST/HIV e que, neste momento, não há eficácia comprovada da $A E$ e dos ARV, portanto, eles não serão prescritos ${ }^{(10-13)}$. A quimioprofilaxia para DST e coleta de sorologia será prescrita no seguimento ambulatorial.

2. Fazer teste rápido de âHCG na urina se houver suspeita de gravidez. Se positivar, encaminhar ao serviço social para receber orientação sobre o protocolo de interrupção legal da gestação(7).

3. Solicitar consulta médica ginecológica imediata na presença de sinais importante de infecção.

4. Acolher familiares e realizar demais orientações descritas no atendimento imediato.

Seguimento Ambulatorial (após uma semana do atendimento inicial, durante seis meses):

1. Investigar sintomas de intolerância aos ARV e orientar sobre alimentação adequada e não ingestão dos medicamentos em jejum para redução dos sintomas(11). 
2. Adequar horário da ingestão do ARV de acordo com a rotina, para melhorar a adesão ao tratamento ${ }^{(11)}$.

3. Reforçar a orientação de hiper-hidratação.

4. Investigar uso de medicamentos que interagem com os $A R V^{(11)}$.

5. Fazer teste rápido de âHCG na urina se houver suspeita de gravidez ${ }^{(9)}$.

6. Reforçar sinais e sintomas de infecção geniturinária.

7. Aferir resultado da primeira sorologia coletada, se negativo, informar o resultado. Qualquer alteração no resultado sorológico (positivo ou não conclusivo) encaminhar para infectologista.

8. Solicitar consulta imediata com médico infectologista e/ou ginecologista se houver sinais importantes de intolerância ou intoxicação química por ARV, de infecção geniturinária ou gravidez.

9. Oferecer, estimular e intervir para o seguimento psicológico nos casos de alteração emocional/sexual e encaminhar ao serviço social se identificar problema econô$\mathrm{mico} / \mathrm{social}$.

10. Reforçar uso do preservativo (fornecer até o retorno), atentando para sinais de disfunção sexual.

11. Reforçar uso de MAC.

12. Informar sobre a rotina do seguimento ambulatorial com equipe multidisciplinar.

13. Orientar sobre exame ginecológico, coleta de secreção vaginal e de sorologias para HIV, hepatite B e C e sífilis, durante 0 atendimento (45, 90 e 180 dias) ${ }^{(10-11)}$.

14. Orientar família/pessoa significativa para o apoio diário. Solicitar o comparecimento ao serviço se julgar necessário.

15. Agendar retorno com enfermeira, ginecologista e infectologista.

\section{Interrupção legal da gestação decorrente de estupro}

No atendimento na gravidez decorrente de estupro a orientação deste processo é realizada pela assistente social, posteriormente, a mulher é atendida pela psicóloga, enfermeira e médico. Se houver solicitação de interrupção da gravidez, ela será discutida em reunião multidisciplinar com a participação da diretoria clínica e da comissão de ética do hospital. A decisão favorável somente será tomada se forem atendidos todos os fatores clínicos (idade da gestação inferior a 20 semanas), psicológicos e os requisitos da legislação brasileira. Se for desejo da mulher continuar com a gravidez, ela é acompanhada por equipe especializada e se optar por doação da criança, ela receberá auxilio para as providências necessárias ${ }^{(7)}$.
0 atendimento de enfermagem é realizado em três momentos distintos: pré-internação, internação e pós-alta hospitalar.

\section{Atendimento ambulatorial - Pré-internação}

1. Acolher, reforçar o desejo e apoiar a decisão de interrupção da gravidez. No caso de dúvida em interromper a gestação, rediscutir com a equipe multidisciplinar.

2. Reforçar a orientação de que, ela ou o representante legal, poderá ser responsabilizado criminalmente caso as declarações sejam falsas ${ }^{(11)}$.

3. Dar apoio emocional e acolher os familiares para que ofereçam apoio no convívio diário após alta hospitalar.

4. Informar sobre os procedimentos de internação.

5. Orientar sobre: coleta de exames, jejum, indução ao aborto, período estimado de internação, curetagem e possíveis riscos cirúrgicos.

\section{Atendimento na unidade de internação}

1. Monitorar sangramento via vaginal.

2. Identificar e intervir no controle da dor.

3. Proporcionar conforto e segurança, durante o período de indução ao aborto.

4. Orientar sobre a coleta e de sorologias para HIV, sífilis e hepatite B e C.

5. Orientar sobre MAC.

6. Orientar sobre o uso de preservativo durante o período de seguimento ambulatorial.

7. Gerenciar a equipe de enfermagem, na manutenção de atitudes sem preconceito ou julgamento, diante da escoIha da mulher em interromper a gestação.

8. Agendar e orientar retorno com enfermeira ambulatorial após sete dias da alta hospitalar.

\section{Atendimento ambulatorial - Após alta hospitalar}

Realizar intervenções descritas no seguimento ambulatorial.

\section{CONSIDERAÇÕES FINAIS}

O Protocolo de Atendimento de Enfermagem às Mulheres Vítimas de Violência Sexual tem proporcionado à cliente um atendimento integral e humanizado e à enfermeira, maior autonomia na sua área de atuação, favorecendo o trabalho colaborativo e interativo com a equipe multidisciplinar.

A experiência de criação e implantação do programa de assistência às mulheres vítimas de violência sexual 
tem influenciado o ensino de graduação e pós-graduação de enfermagem da Unicamp. Essa temática é discutida em sala de aula dentro da abordagem da violência de gênero, buscando sensibilizar os(as) alunos(as) quanto a essa questão, por meio de reflexões sobre os fatores históricos, culturais e sociais que contribuem para a manuten-

\section{REFERÊNCIAS}

1. Heise L. Gender-based abuse: the global epidemic. Cad Saúde Pública. 1994;10 Supl 1:135-45.

2. Brasil. Ministério da Saúde. Secretaria de Políticas de Saúde. Departamento de Gestão de Política Estratégicas. Área Técnica Saúde da Mulher. Normas sobre a prevenção e tratamento dos agravos resultantes da violência sexual contra mulheres e adolescente. Brasília; 1999.

3. Hoga LAK. A dimensão subjetiva do profissional na humanização da assistência a saúde: uma reflexão. Rev Esc Enferm USP. 2004;38(1):13-20.

4. Hatmaker DD, Pinholster L, Saye JJ. A community-based approach to sexual assault. Public Health Nurs. 2002;19 (2):124-7.

5. Stermac LE, Stirp TS. Efficacy of a 2-year-old sexual assault Nurse Examiner Program in Canadian Hospital. J Emerg Nurs. 2002;28(1):18-23.

6. Campinas. Secretaria Municipal de Saúde. Coordenadoria da Mulher. Iluminar Campinas - Cuidando das Vítimas de Violência Sexual [texto na Internet]. [citado 2006 abr. 2]. Disponível em: http://antigo. campinas.sp.gov.br/ saude/programas/iluminar/iluminar_textobase.htm

7. Maia CAT, Mondaca ADCA, Duarte CS, Lima JA, Colodo M, Lemos TM, et al. Mulheres vítimas de violência sexual: atendimento multidisciplinar. Femina. 2000;28(1):155-61.

8. SOS Ação Mulher e Família: histórico [texto na Internet]. Campinas; 2004. [citado 2004 abr. 2]. Disponível em: http:// www. preac.unicamp.br/sosacaomulher/html/historico.htm

9. World Health Organization (WHO). Guidelines for medico-legal care for victims of sexual violence. Geneva; 2003. ção deste tipo de violência em nosso meio. As experiências do SOSAção Mulher e Família e do Caism têm contribuído para a formação dos(as) futuros(as) enfermeiros(as) a medida em que mostram o que se pode fazer e o que ainda é preciso ser feito para que a sociedade seja mais humana e justa para com as mulheres.

10. World Health Organization (WHO). Clinical management of rape survivors: developing protocols for use with refugee and internally displaces persons. Geneva; 2004.

11. Brasil. M inistério da Saúde. Secretaria de Políticas de Saúde. Departamento de Gestão de Política Estratégicas. Área Técnica Saúde da Mulher. Normas sobre a prevenção e tratamento dos agravos resultantes da violência sexual contra mulheres e adolescente. Brasília; 2005.

12. World Health Organization (WHO). Emergency contraception: a guide for service delivery; WHO/FRH/FPP/ 98.19. Geneva; 1998

13. Brasil. Ministério da Saúde. Secretaria de Atenção à Saúde. Departamento de Ações Programáticas Estratégicas. Área Técnica Saúde da Mulher. Anticoncepção de emergência: perguntas e respostas para profissionais de saúde. Brasília; 2005.

14. Braga MFC, Moura MAV. A atuação da enfermeira no cuidado à mulher em situação de violência sexual. In: Livro-Temas do 55 Congresso Brasileiro de Enfermagem; 2004 out. 24-29; Gramado, BR. [evento na Internet]. Gramado; 2004. [citado 2006 jan. 23]. Disponível em: http://www.bstorm.com.br/enfermagem/index-xp2. php? cod $=61456 \&$ popup $=1$

15. North American Nursing Diagnosis Association (NANDA). Diagnósticos de enfermagem da NANDA: definições e classificações 2003-2004. Trad. de Cristina Correa. Porto Alegre: Artmed; 2005. 